\title{
Characterization of Sedentary Behavior in Heart Failure Patients With Arthritis
}

\author{
Qi Zhang ${ }^{\mathrm{a}}$, Mark Schwade ${ }^{\mathrm{b}}$, Pascha Schafer ${ }^{\mathrm{b}}$, Neal Weintraub ${ }^{\mathrm{b}}$, Lufei Young ${ }^{\mathrm{c}, \mathrm{d}}$
}

\begin{abstract}
Background: Arthritis is one of the most common comorbidities in heart failure (HF) patients, and is associated with decreased activity levels. Few studies have examined sedentary behavior (SB) in HF patients with arthritis, and little is known about the factors that may influence SB in this population.
\end{abstract}

Methods: This is a retrospective, secondary analysis using data collected from a randomized control trial. SB was measured by the daily sedentary time collected by accelerometers. Structural equation modeling was performed to examine relationships between key concepts based on social cognitive theory, and elucidate the potential pathways by which demographic, clinical and sociobehavioral factors that influence SB.

Results: A total of 101 participants' data were used for this analysis. Participants were mainly female $(\mathrm{n}=64,63 \%)$ with a mean age of 70 years (standard deviation $(\mathrm{SD})=12.2$ ) and an average of 13 years of education $(\mathrm{SD}=2.3)$. SB was highly prevalent at baseline (mean value: $21.0 \mathrm{~h} /$ day), 3 months (mean value: $20.6 \mathrm{~h} /$ day) and 6 months (mean value: $20.8 \mathrm{~h} /$ day) in study participants. Factors with statistically significant positive association with sedentary time include age and retirement, while significant negative association was found with current employment. HF self-care efficacy and behavior were also significantly associated with SB.

Conclusions: Most HF patients with arthritis in this study lived a sedentary lifestyle. Additional studies are needed to identify feasible and effective exercise programs for HF participants with arthritis.

Keywords: Sedentary behavior; Heart failure; Arthritis; Structural equation modeling

Manuscript submitted January 22, 2020, accepted February 3, 2020

aThe Nethersole School of Nursing, Faculty of Medicine, The Chinese University of Hong Kong, Hong Kong, China

bepartment of Medicine, Medical College of Georgia at Augusta University, Augusta, GA, USA

${ }^{\mathrm{c} C o l l e g e}$ of Nursing, Augusta University, Augusta, GA, USA

${ }^{\mathrm{d} C}$ Corresponding Author: Lufei Young, College of Nursing, Augusta University, 987 St. Sebastian Way, Augusta, GA 30912, USA.

Email: luyoung@augusta.edu

doi: https://doi.org/10.14740/cr1023

\section{Introduction}

Sedentary behavior (SB) is defined as any waking behavior characterized by energy expenditure $\leq 1.5$ metabolic equivalents (METs) while in a sitting or reclining posture [1]. SB has become the major risk factor for cardiovascular diseases, associated with significant disability and mortality in patients with heart failure (HF) [2]. It has been reported that SB in patients with $\mathrm{HF}$ is associated with nearly twice the risk of cardiac death and all-cause mortality [3-5]. In addition, one of the most common comorbid conditions among patients with HF is arthritis, which appears to be a major contributor to physical inactivity and SB [6]. The burden of HF is even greater among rheumatoid arthritis patients [7-9], who experience a two-fold higher HF incidence rate in comparison with general population [7]. HF patients with arthritis are $60 \%$ more likely to live a sedentary life $[5,10,11]$. Furthermore, mortality following HF is significantly higher in patients with rheumatoid arthritis [12]. Therefore, increased SB may be a modifiable independent risk factor of health outcomes in HF patients with arthritis. $\mathrm{SB}$ is often measured by self-report questionnaires, which is a less valid and reliable method when compared to objective measures [13]. Inaccurate measurements of SB make it difficult to evaluate the intervention effects and monitor changes in $\mathrm{SB}$ over time. In contrast, objective measures, such as accelerometers, are believed to offer more accuracy without recall and response bias which exist in self-reported measures [14]. The accelerometer is the most widely used and accepted objective measure of daily physical activity (PA) and calorie expenditure in research and clinical settings [15].

Accelerometry uses triaxial measurements of movement in three orthogonal directions to report estimates of both intensity and duration of bodily movements [13], and has been used to gather detailed information about SB in various patient/ healthy populations [16]. However, the use of accelerometery to assess SB in HF has been rarely reported. Furthermore, the factors influencing SB and subsequent PA levels in HF have yet to be fully elucidated [17]. In general, social cognitive theory (SCT) has been one of the predominant theoretical frameworks to guide the study in PA [18-20], however little is known about the process through which SCT variables influence SB in HF patients with arthritis [21]. To develop and implement more effective interventions to reduce SB in HF patients with arthritis, a better understanding of the factors underlying SB is needed. Therefore, the purpose of this study was to test an SCT model of PA, created by hypothesizing direct and indirect 
effects of demographic, clinical, and sociobehavioral factors on SB among HF patients with arthritis.

\section{Materials and Methods}

This study was a secondary analysis using the data from a randomized controlled trial aimed at examining the effectiveness of a 12-week home-based intervention to improve HF selfmanagement adherence [22]. The original/parent study was approved by the University Institutional Review Board and the rural hospital ethics committee. All participants of the parent study gave written informed consent [22]. Taking a different focus from the original study, the purpose of this secondary analysis was to describe SB measured by accelerometers and examine the factors influencing SB in HF patients with arthritis.

Participants were recruited from the hospital, and eligible participants: 1) were aged 21 years or older; 2) had HF as one of their discharge diagnoses; 3) were classified as New York Heart Association (NYHA) class II or above, and had at least one HF-related hospitalization or emergency department visit in the previous year; 4) with an established diagnosis of arthritis; 5) were discharged to home; 6) passed a mini-cog screen test [23]; 7) understood English; and 8) had access to a phone. We excluded participants who: 1) had depressive symptoms, and received a score of 3 or above on the Participant Health Questionnaire-2 (PHQ-2) [24]; 2) were diagnosed with liver cirrhosis; 3) were diagnosed with chronic renal failure; or 4) were diagnosed with other end-stage and/or terminal illness (e.g., cancer), which limited the participant's ability to perform moderate or above PA. The study setting has been described in more detail in a previously published manuscript [22].

Socio-demographic characteristics of the study subjects were assessed by using a previously utilized questionnaire [22] that asked subjects about their gender, age, years of school, marital status, annual household income, and employment status. Blood samples were drawn for analysis of plasma brain natriuretic peptide (BNP). Other clinical data (e.g., laboratory results, echocardiographs, medical history, and prescribed medications) were also collected from the medical records at baseline.

The primary outcome, SB, was defined as the average time spent in sedentary physical activity (SED-time) per day, which is measured by the accelerometer and classified as anything under 1.5 METS [25]. All patients were instructed to wear an ActiGraph GT3X+ device (GT3X; Actigraph, Pensacola, FL, USA), to assess SED-time and daily PA. The accelerometer was attached to an elastic belt around the waist and positioned on the non-dominant hip [26] for a minimum of $8 \mathrm{~h}$ a day for 7 consecutive days, as previously recommended [27, 28]. According to manufacture specifications, the accelerometer was initiated through ActiLife v6 (ActiGraph LLC, Pensacola, FL, USA) at a sampling rate of $60 \mathrm{~Hz}$ [29]. The Freedson algorithm [30] was used to quantify wake and sleep periods. After being downloaded in ActiLife software, data were converted into vector magnitude (VM) counts at 60-s epochs. The Freedson combination (1998) energy expenditure formula was used to compute daily activity calories, and the Freedson adult

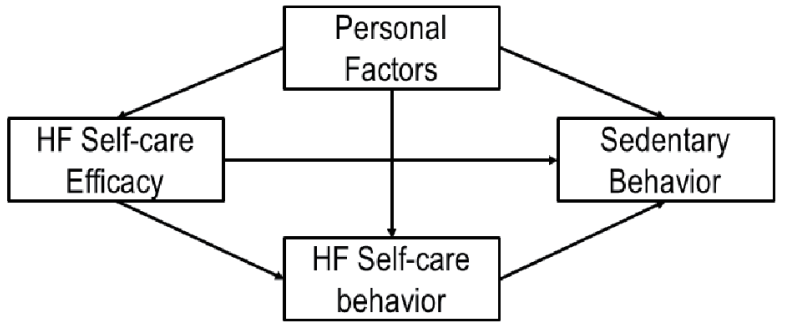

Figure 1. Proposed conceptual framework of factors related to sedentary behavior in HF patients. HF: heart failure.

(1998) cut point classification was used to determine daily minutes spent on sedentary intensity ( 0 - 99 counts per minutes), light intensity (100 - 759 counts per minutes), lifestyle intensity (760 - 5,724 counts per minutes), moderate intensity (1,952 - 5,724 counts per minutes), vigorous intensity $(5,725$ 9,498 counts per minutes) and very vigorous intensity $(>9,499$ counts per minutes) physical activities [31].

To examine the factors related to SB, we assessed HF selfcare knowledge, HF self-care self-efficacy, and HF self-care behavior. Based on the social cognitive theory, the proposed conceptual framework is presented in Figure 1. HF self-care knowledge was measured with Atlanta HF knowledge test (AHFKT-V2), 27-item multiple-choice questions to measure HF self-management knowledge [32]. The latent variable, HF self-care self-efficacy was measured by Self-Care of Heart Failure Index [33]. HF self-care behavior was assessed by using 29-item Revised Heart Failure Self-Care Behavior Scale (RSCB) [34].

Data are expressed as mean \pm standard deviation (SD) or number of cases (percentage). Unpaired Student's $t$-tests and $\chi^{2}$ test were used for comparisons of continuous and categorical variables, respectively. Mann-Whitney U-test was utilized to compare data that were not normally distributed. Pearson and Spearman correlation coefficients were used to identify the relationship between variables associated with SB. Partial correlation was also performed by adjusting each bivariate correlation for confounders. Then, multivariate regression analysis, with stepwise backward selection of variables, was used to determine the independent correlates of SB. The Akaike's information criterion was used to determine the most appropriate predictive model [35]. For all crossover end points, the analysis was performed on an intention-to-treat basis. A P value $<0.05$ was considered statistically significant. All statistical analyses were performed using IBM SPSS for windows software (SPSS Inc., Chicago, IL, USA).

Structural equation modeling (SEM) was used to study associations between a series of mediating relationships because it allows analysis of a variable's direct, indirect, and total effects simultaneously, and it elucidates the potential pathways by which demographic, clinical and sociobehavioral factors influence SB. Missing data were handled by approximating the means, and the maximum-likelihood estimation procedure was applied. Four fit indexes were used, non-normed fit index (NNFI), comparative fit index (CFI) [36], root mean square error of approximation (RMSEA) [37], and the standardized root mean residual (SRMR). Acceptable model fit is generally 
based on standard fit index criteria (NNFI $>0.90$, CFI $>0.90$, RMSEA $<0.08$, SRMR $<0$.08). SEM was done using the software IBM Amos.

\section{Results}

A study sample of 101 participants entered the analysis. The baseline characteristics of the study population are presented in Table 1. Sub-group descriptive statistics were also performed by gender and body mass index (BMI) (Table 1). Participants' mean age was $70(\mathrm{SD}=12.2)$ years. Majority of participants were female $(63 \%)$, white $(95 \%)$, retired $(71 \%)$, and an average of $13(\mathrm{SD}=2.3)$ years of education. Most participants' cardiac functioning was classified at NYHA level II (49\%) or III $(42 \%)$, with a preserved ejection fraction $(55.7 \pm 11.1 \%)$. Participants had an average BMI of $32.3 \mathrm{~kg} / \mathrm{m}^{2}(\mathrm{SD}=7.1)$, and $54.46 \%$ of them was overweight $(\mathrm{BMI} \geq 30$ ).

The average SED-time measured by the accelerometer was $1,262 \mathrm{~min} /$ day $(21 \mathrm{~h} /$ day $)$ at baseline, $1,237 \mathrm{~min} /$ day $(20.6 \mathrm{~h} /$ day $)$ at 3 months, and 1,245 $\mathrm{min} /$ day $(20.8 \mathrm{~h} /$ day $)$ at 6 months (Table 2 and Fig. 2). In the subgroup analysis by sex, men had slightly higher but not statistically significant SED-time compared to women at all time points (Fig. 3). The subgroup analysis by BMI showed no difference in SED-time between obese and non-obese at baseline, 3 or 6 months. The SED-time at baseline, 3 and 6 months by sex and BMI is presented in Table 2.

At baseline, HF patients with arthritis spent an average of $89 \%$ of the day in SB, $9.5 \%$ in light intensity PA level, $1.5 \%$ in lifestyle activity level, and less than $0.2 \%$ in moderate and vigorous PA level of PA.

The bivariate correlation showed negative relationships between SED-time and being employed, levels of blood hemoglobin (HGB), hematocrit (HCT), and dietary sodium intake that was assessed by $24 \mathrm{~h}$ urinary sodium excretion (Table 3 ). In multiple regression models, SED-time was negatively associated with self-care knowledge, HGB, and systolic blood pressure at hospital admission, while positively associated with log transformed BNP, white blood cell and discharge weight (Table 4). NYHA HF classification was also included in the bivariate analysis and was therefore controlled for and found not to be significantly associated with SED time $(\mathrm{P}=0.25)$.

The SEM was used to develop and test the proposed model underpinned by the social cognitive theory (Fig. 4). The model-fit statistics was acceptable (CFI $=0.947$, AGFI $=$ 0.908 , RMSEA $=0.001)$. The personal factors entered in the final model included age, employment status, and HGB. Employment status was significantly related to SED-time (standardized coefficient ( $\mathrm{r}$ ) was $0.36, \mathrm{P}<0.001)$. Age was positively related with HF self-care behavior measured by $\mathrm{RSCB}(\mathrm{r}=$ $0.26, \mathrm{P}<0.01)$. There was a nearly significant negative correlation between HGB and SED-time $(r=0.18, \mathrm{P}=0.06)$.

Among the three SCT-derived concepts (i.e., self-care knowledge, self-care efficacy and self-care behavior), self-care knowledge was not significantly associated with SED-time or other concepts of interest. HF self-care behavior measured by RSCB was associated with a decrease in SED-time $(\mathrm{P}=0.01)$. HF self-care efficacy was not directly related to SED-time, however, self-care efficacy was related to self-care behavior $(\mathrm{r}=0.39, \mathrm{P}<0.01)$. On the other hand, self-care efficacy was indirectly related to SED-time, the higher HF self-care efficacy was associated with the decreased SED-time. Therefore, HF self-care behavior mediates the effect of HF self-care efficacy on SED-time.

\section{Discussion}

To our knowledge, this is the first study describing the prevalence of SB among HF patients with arthritis using accelerometry to objectively measure PA. In addition, we also examined the factors influencing SB in this population based on Bandura's Social Cognitive Theory (SCT). As far as we know, this is first study that reported a conceptual framework of factors influencing SB based on our structure equation modeling results, which may direct researchers and clinicians to develop effective interventions to reduce $\mathrm{SB}$ and promote $\mathrm{PA}$ in $\mathrm{HF}$ patients with arthritis.

In previous studies, SB was often measured with self-report questionnaire $[5,38]$, which is often invalid, unreliable, and fails to provide the detailed information about SB pattern. Without accurate and appropriate PA measurements, it would be challenging to design interventions to promote PA and evaluate their effects. In contrast, accelerometers are more accurate and provide detailed information about the pattern of SB. Consistent with previous evidence [39], SB is highly prevalent in HF patients.

Based on our SEM analysis, we developed a theoretical framework to explain factors influencing SB among HF patients with arthritis. Our SEM analysis highlights the inverse association between SED-time and employment status. Those who have a full-time or part-time job may be more likely to live a healthier and perhaps somewhat more active life. These findings extend previous research that employment status is related to PA and SED-time among HF patients with arthritis [40]. Our study confirms findings from previous studies that those participants currently working, especially on weekdays [41], were less sedentary and more active than non-employed. HF patients with arthritis could be targeted with programs to increase employment as to decrease SB.

In addition, our conceptual framework illustrates the relationships between SB and two main concepts proposed in Bandura's SCT; self-efficacy and self-care behavior. Our model demonstrates that self-care behavior mediates the impact of self-care efficacy on SB in HF patients with arthritis. The patients with greater confidence in self-care were more likely to engage in self-care activities, therefore, less likely to live a sedentary lifestyle. Those with part-time or full-time jobs were more likely to be active, while the self-care activities diminished with advancing age. SB was also affected by the HGB concentration. Patients with lower HGB are less likely to be active, which is consistent with others' findings, potentially due to increased fatigue $[15,42]$.

Several limitations exist in this study. First, the study used a convenient sample; therefore, caution should be used in generalizing the results to other HF patients without arthritis. Secondly, although objective measures can provide precise time 


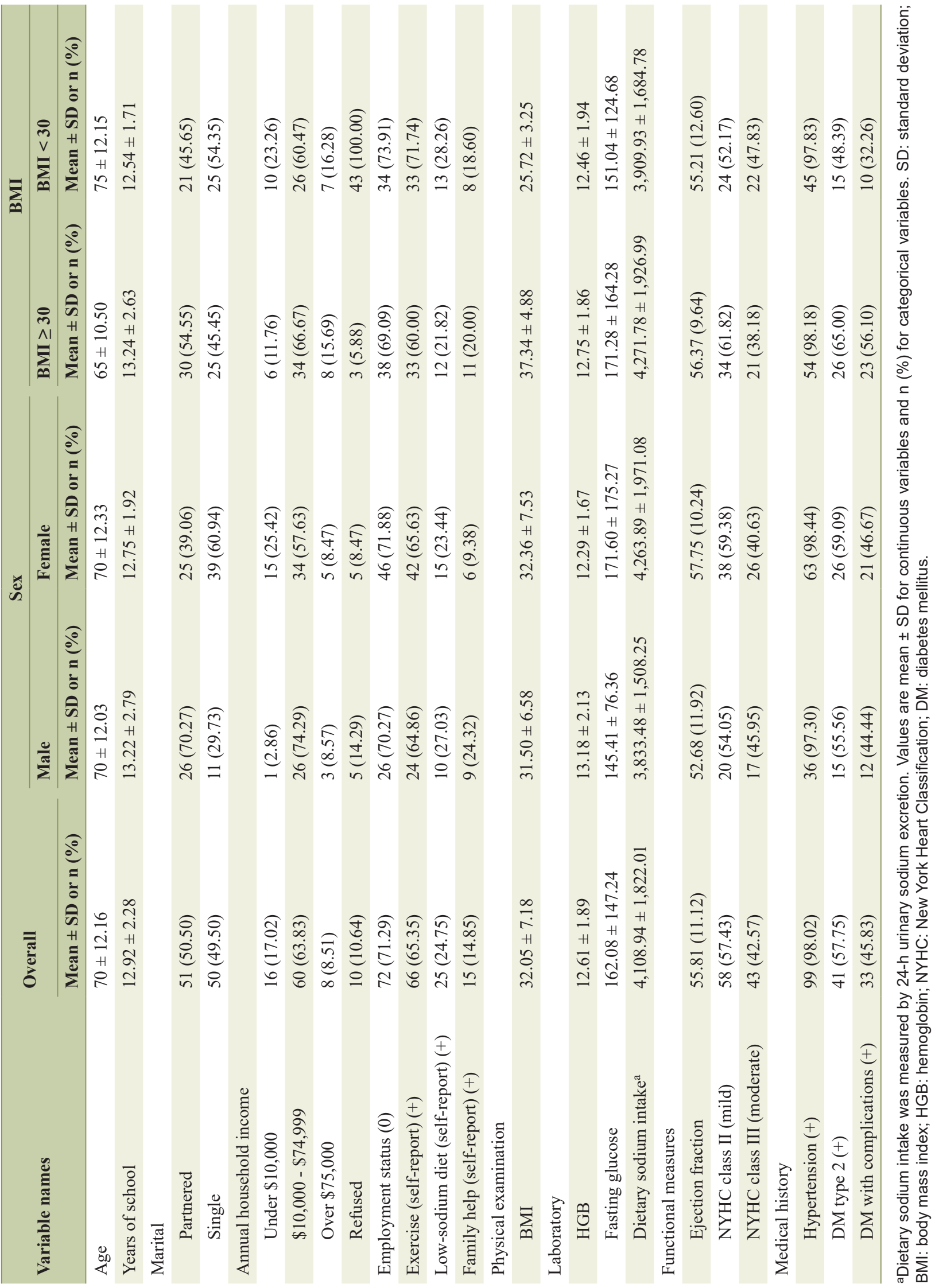



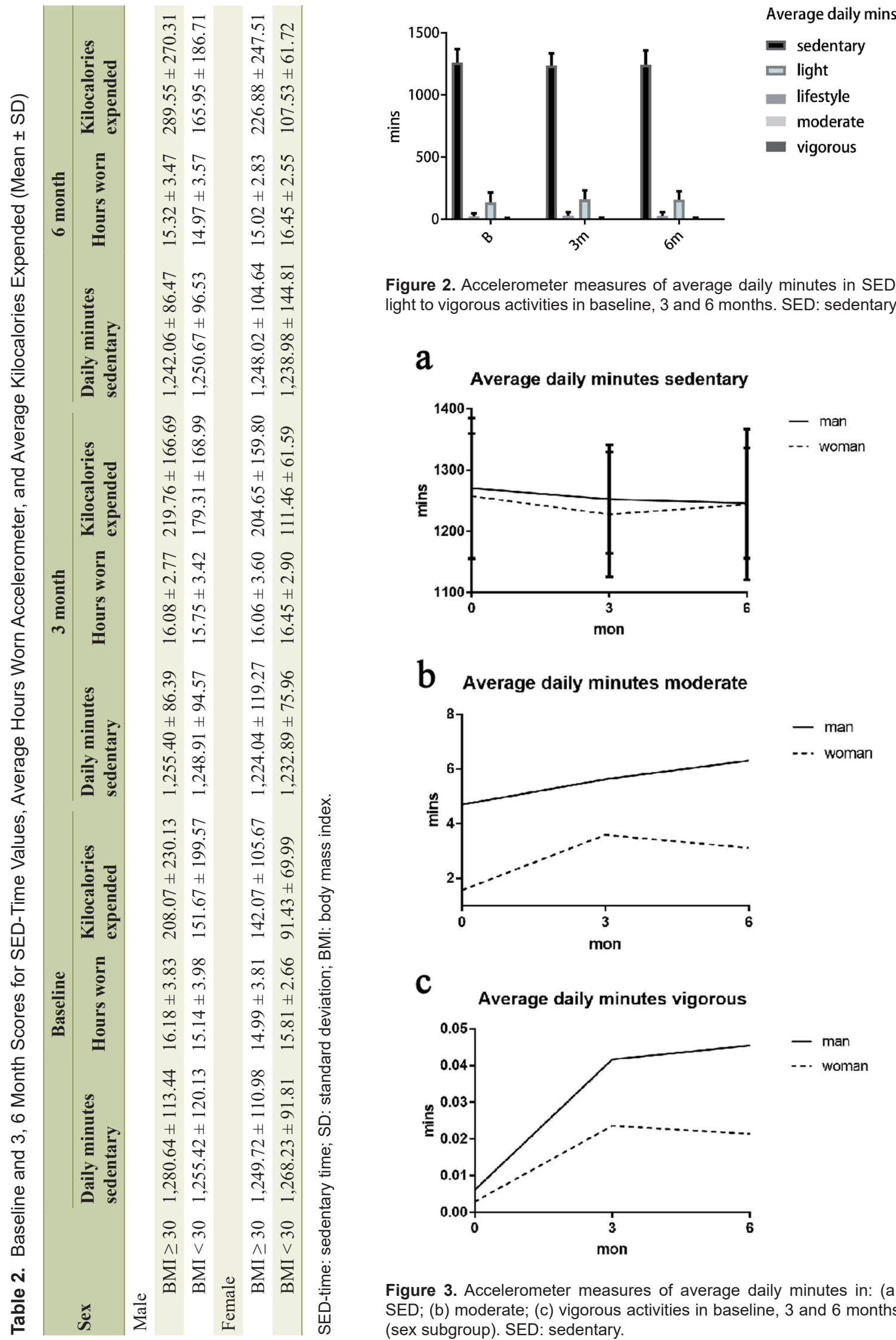

Figure 2. Accelerometer measures of average daily minutes in SED, light to vigorous activities in baseline, 3 and 6 months. SED: sedentary.

a

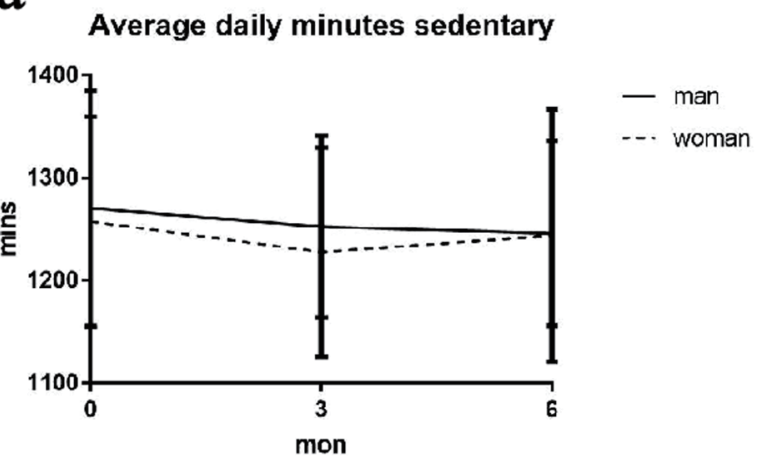

b Average daily minutes moderate
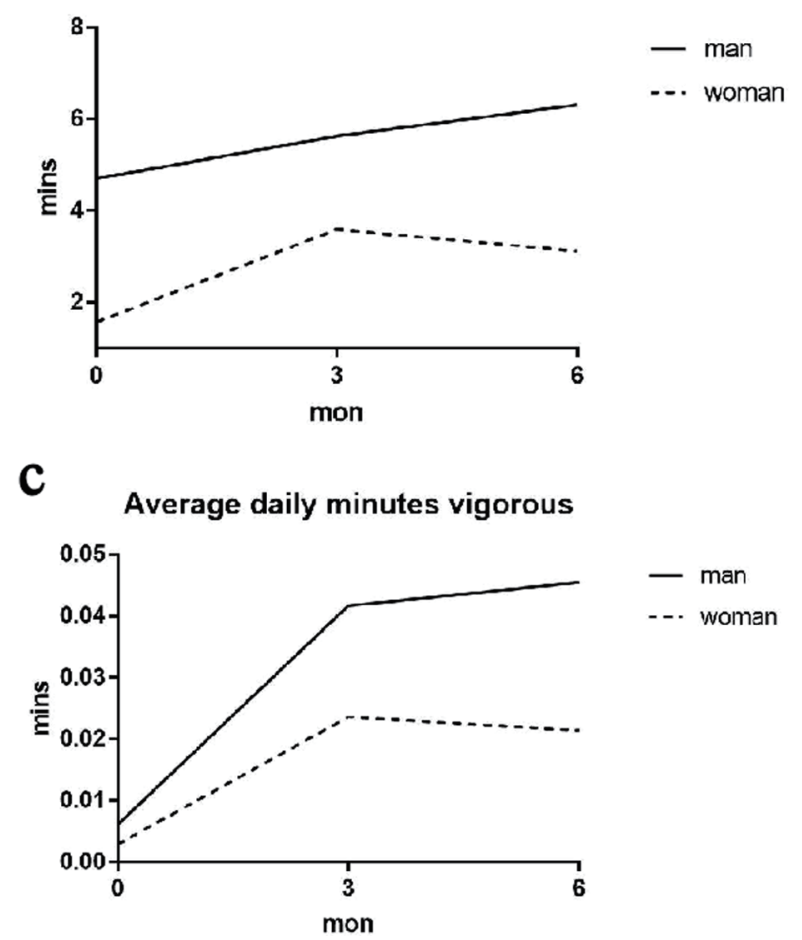

Figure 3. Accelerometer measures of average daily minutes in: (a) SED; (b) moderate; (c) vigorous activities in baseline, 3 and 6 months (sex subgroup). SED: sedentary. 
Table 3. Bivariate Correlations of Sedentary Time With Potential Parameters

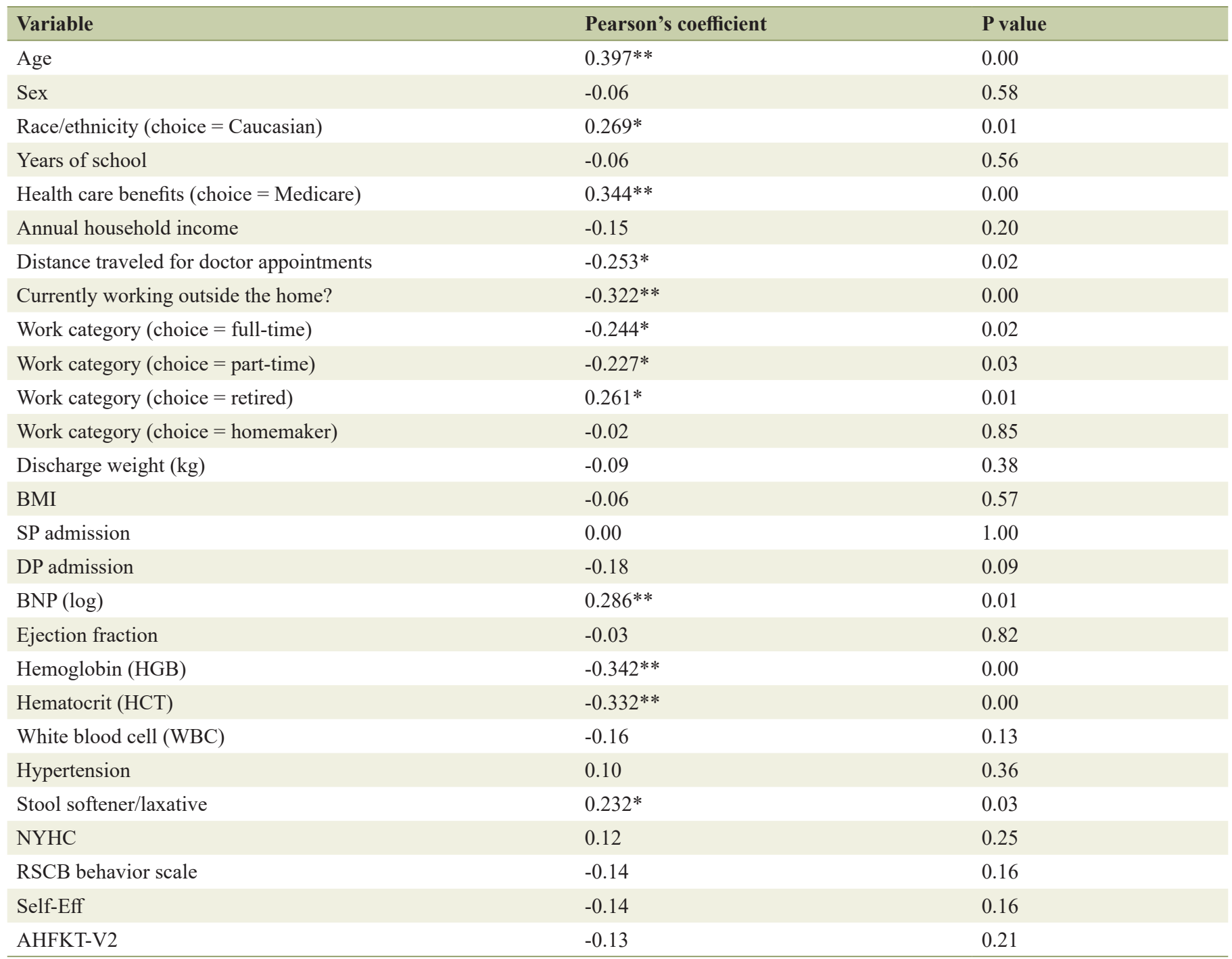

${ }^{* *} \mathrm{P}<0.01,{ }^{*} \mathrm{P}<0.05$. BMI: body mass index; SP: systolic pressure; DP: diastolic blood pressure; BNP: B-type natriuretic peptides; NYHC: New York Heart Classification; RSCB: 29-item Revised Heart Failure Self-Care Behavior Scale; Self-Eff, Self-Efficacy for HF Self-management Measure by Self-Care of Heart Failure Index; AHFKT: Atlanta heart failure knowledge test (AHFKT-V2).

Table 4. Multiple Regression Analysis of Average Daily Minutes of SED

\begin{tabular}{lll}
\hline Variable & $\boldsymbol{\beta}$ & P \\
\hline Medical knowledge & -0.629 & 0 \\
Hemoglobin (HGB) & -0.446 & 0 \\
BNP & 0.559 & 0 \\
SP admission & -0.326 & 0.005 \\
White blood cell (WBC) & 0.339 & 0.003 \\
Discharge weight $(\mathrm{kg})$ & 0.233 & 0.035 \\
\hline
\end{tabular}

Multiple regression analysis with stepwise backward selection of variables; Determinants: average daily minutes of sedentary. Final model $(n=64)$. $\beta$ : beta coefficient; BNP: B-type natriuretic peptide; SP: systolic pressure. and accurate individual behavioral level estimates, the information on "what, how, when and where" the individual was doing in real-world is unavailable. Third, waist-worn accelerometers are unable to accurately detect activities of upperbody or lower-body movements, thus it is possible that actual SED-time may be overestimated. Finally, this study did not control for patient's severity of arthritis, which could influence patient's SED-time.

Findings of the present study have important clinical implications. First, this is the first study to examine the factors that contribute to SB in HF patients with arthritis using objective data collected from an accelerometer. Evidence has shown that HF patients with arthritis have greater challenges staying physically active, supporting the need for targeted interventions to promote PA in this vulnerable population. Second, the developed conceptual model has implications for future re- 


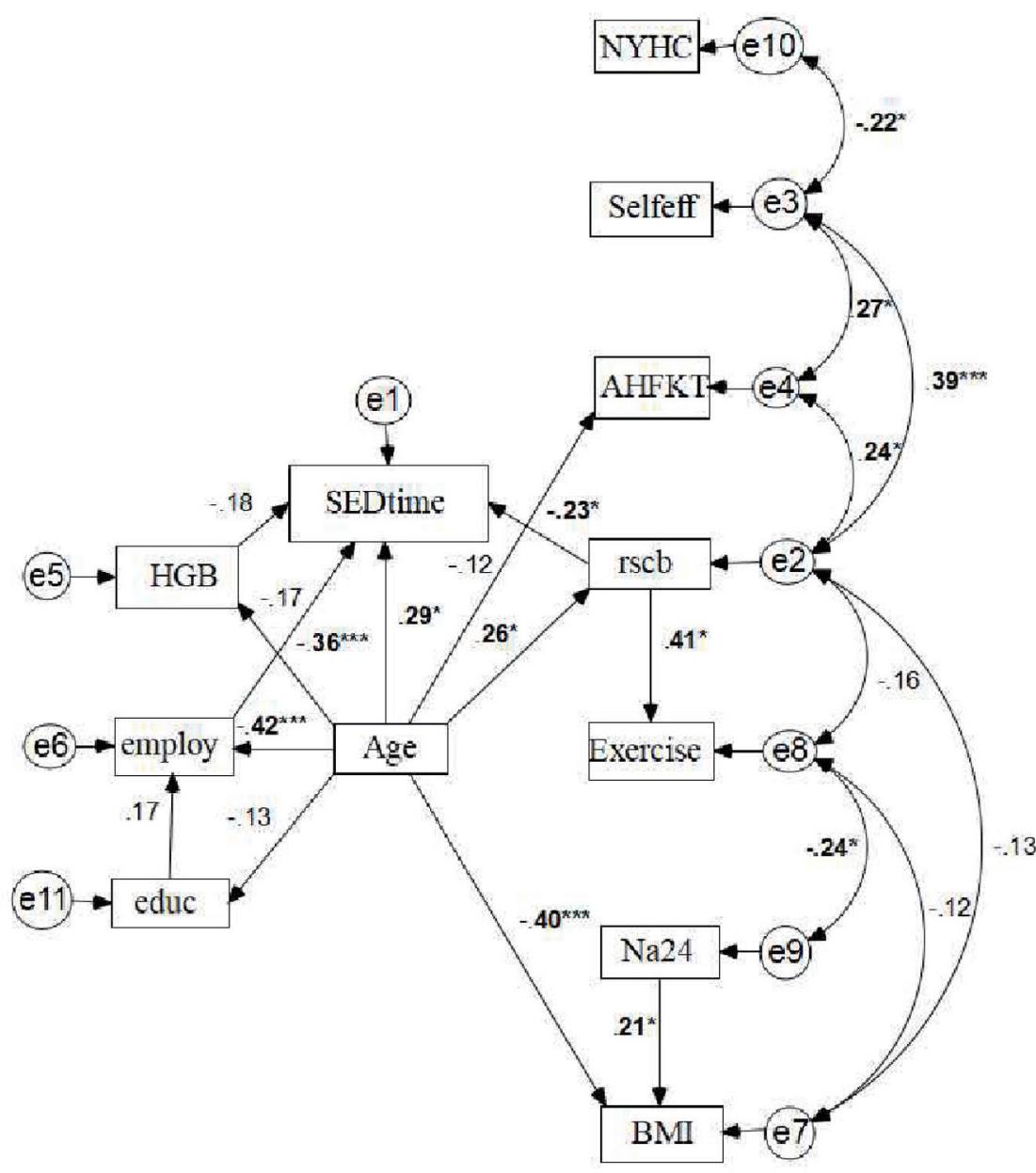

Figure 4. Standardized parameter estimates for proposed theoretical model based upon SCT. * $P<0.05$. ${ }^{* *} P<0.001$. Rectangles represent observed variables, independent variable with the arrow pointing toward dependent variable. The line with two arrows represents a possible correlation between the two variables, the e1 - e11 in circle represent error terms; while the numbers adjacent to the lines are standardized direct effects between the pair of connected variables. SED-time: sedentary time; HGB: hemoglobin; Educ: education; NYHC: New York Heart Classification; Self-Eff: Self-Efficacy for HF Self-management Measure by Self-Care of Heart Failure Index; AHFKT: Atlanta heart failure knowledge test (AHFKT-V2); RSCB: 29-item Revised Heart Failure Self-Care Behavior Scale; Na24: 24-h urinary Na; BMI: body mass index.

search and practice. The model will be valuable in identifying and targeting patients at high risk to develop tailored strategies to reduce barriers, reduce SB, and promote PA.

\section{Conclusions}

In conclusion, SB in HF patients with arthritis is highly prevalent. Furthermore, SCT was considered an appropriate theoretical framework, and there is a strong correlation between SEDtime and patient's employment status, HF self-care behavior, self-efficacy, and age. Our conceptual model may be helpful in designing specific interventions to reduce SB and promote PA among HF patients with arthritis. Further study is necessary to compare SED-time in HF patients with and without arthritis to determine how our results are generalizable to the entire HF population.

\section{Acknowledgments}

None to declare.

\section{Financial Disclosure}

This research was funded by National Institute of Health and National Institute of Nursing Research (NIH/NINR) through grant number 1R15NR 13769-01A1.

\section{Conflict of Interest}

There are no conflicts of interest, including no financial conflicts of interest for all authors of this manuscript. 


\section{Informed Consent}

Written informed consents were obtained from all participants of the parent study.

\section{Author Contributions}

L. Young conducted the original study; Q. Zhang performed this secondary data analysis and the development of original manuscript. Q. Zhang, L. Young, and M. Schwade contributed to writing of the manuscript, as well as critically analyzing the content and improving the quality of the manuscript. P. Schafer and $\mathrm{N}$. Weintraub verified the analytical methods, findings, and reviewed revisions. All authors discussed the results and contributed to the final versions of the manuscript.

\section{Data Availability}

The data supporting the findings of this study are available from the corresponding author upon reasonable request. Any inquiries regarding supporting data availability of this study should be directed to the corresponding author.

\section{References}

1. Stamatakis E, Ekelund U, Ding D, Hamer M, Bauman $\mathrm{AE}$, Lee IM. Is the time right for quantitative public health guidelines on sitting? A narrative review of sedentary behaviour research paradigms and findings. Br J Sports Med. 2019;53(6):377-382.

2. Lavie CJ, Ozemek C, Carbone S, Katzmarzyk PT, Blair SN. Sedentary behavior, exercise, and cardiovascular health. Circ Res. 2019;124(5):799-815.

3. Park LG, Dracup K, Whooley MA, McCulloch C, Lai S, Howie-Esquivel J. Sedentary lifestyle associated with mortality in rural patients with heart failure. Eur J Cardiovasc Nurs. 2019;18(4):318-324.

4. Jefferis BJ, Parsons TJ, Sartini C, Ash S, Lennon LT, Papacosta O, Morris RW, et al. Objectively measured physical activity, sedentary behaviour and all-cause mortality in older men: does volume of activity matter more than pattern of accumulation? Br J Sports Med. 2019;53(16):1013-1020.

5. Doukky R, Mangla A, Ibrahim Z, Poulin MF, Avery E, Collado FM, Kaplan J, et al. Impact of physical inactivity on mortality in patients with heart failure. Am J Cardiol. 2016;117(7):1135-1143.

6. Centers for Disease Control and Prevention. Arthritis as a potential barrier to physical activity among adults with heart disease-United States, 2005 and 2007. MMWR Morb Mortal Wkly Rep. 2009;58(7):165-169.

7. Nicola PJ, Maradit-Kremers H, Roger VL, Jacobsen SJ, Crowson CS, Ballman KV, Gabriel SE. The risk of congestive heart failure in rheumatoid arthritis: a population-based study over 46 years. Arthritis Rheum.
2005;52(2):412-420

8. Van Doornum S, Jennings GL, Wicks IP. Reducing the cardiovascular disease burden in rheumatoid arthritis. Med J Aust. 2006;184(6):287-290.

9. Wolfe F, Michaud K. Heart failure in rheumatoid arthritis: rates, predictors, and the effect of anti-tumor necrosis factor therapy. Am J Med. 2004;116(5):305-311.

10. Legge A, Blanchard C, Hanly JG. Physical activity and sedentary behavior in patients with systemic lupus erythematosus and rheumatoid arthritis. Open Access Rheumatol. 2017;9:191-200.

11. Fenton SAM, Veldhuijzen van Zanten J, Kitas GD, Duda JL, Rouse PC, Yu CA, Metsios GS. Sedentary behaviour is associated with increased long-term cardiovascular risk in patients with rheumatoid arthritis independently of moderate-to-vigorous physical activity. BMC Musculoskelet Disord. 2017;18(1):131.

12. Davis JM, 3rd, Roger VL, Crowson CS, Kremers HM, Therneau TM, Gabriel SE. The presentation and outcome of heart failure in patients with rheumatoid arthritis differs from that in the general population. Arthritis Rheum. 2008;58(9):2603-2611.

13. Falck RS, McDonald SM, Beets MW, Brazendale K, LiuAmbrose T. Measurement of physical activity in older adult interventions: a systematic review. Br J Sports Med. 2016;50(8):464-470.

14. Dyrstad SM, Hansen BH, Holme IM, Anderssen SA. Comparison of self-reported versus accelerometer-measured physical activity. Med Sci Sports Exerc. 2014;46(1):99106.

15. Saint-Maurice PF, Welk GJ, Beyler NK, Bartee RT, Heelan KA. Calibration of self-report tools for physical activity research: the Physical Activity Questionnaire (PAQ). BMC Public Health. 2014;14:461.

16. Pozehl BJ, McGuire R, Duncan K, Hertzog M, Deka P, Norman J, Artinian NT, et al. Accelerometer-measured daily activity levels and related factors in patients with heart failure. J Cardiovasc Nurs. 2018;33(4):329-335.

17. Klompstra L, Jaarsma T, Stromberg A. Physical activity in patients with heart failure: barriers and motivations with special focus on sex differences. Patient Prefer Adherence. 2015;9:1603-1610.

18. Lee CG, Park S, Lee SH, Kim H, Park JW. Social Cognitive Theory and Physical Activity Among Korean Male HighSchool Students. Am J Mens Health. 2018;12(4):973-980.

19. Uszynski MK, Casey B, Hayes S, Gallagher S, Purtill H, Motl RW, Coote S. Social cognitive theory correlates of physical activity in inactive adults with multiple sclerosis. Int J MS Care. 2018;20(3):129-135.

20. Bagherniya M, Mostafavi Darani F, Sharma M, Maracy MR, Allipour Birgani R, Ranjbar G, Taghipour A, et al. Assessment of the Efficacy of Physical Activity Level and Lifestyle Behavior Interventions Applying Social Cognitive Theory for Overweight and Obese Girl Adolescents. J Res Health Sci. 2018;18(2):e00409.

21. Klompstra L, Jaarsma T, Stromberg A. Self-efficacy mediates the relationship between motivation and physical activity in patients with heart failure. J Cardiovasc Nurs. 2018;33(3):211-216. 
22. Young L, Hertzog M, Barnason S. Effects of a homebased activation intervention on self-management adherence and readmission in rural heart failure patients: the PATCH randomized controlled trial. BMC Cardiovasc Disord. 2016;16(1):176.

23. Borson S, Scanlan J, Brush M, Vitaliano P, Dokmak A. The mini-cog: a cognitive 'vital signs' measure for dementia screening in multi-lingual elderly. Int $\mathrm{J}$ Geriatr Psychiatry. 2000;15(11):1021-1027.

24. Lowe B, Kroenke K, Grafe K. Detecting and monitoring depression with a two-item questionnaire (PHQ-2). J Psychosom Res. 2005;58(2):163-171.

25. Aguilar-Farias N, Brown WJ, Peeters GM. ActiGraph GT3X+ cut-points for identifying sedentary behaviour in older adults in free-living environments. J Sci Med Sport. 2014;17(3):293-299.

26. Huberty J, Ehlers DK, Kurka J, Ainsworth B, Buman M. Feasibility of three wearable sensors for 24 hour monitoring in middle-aged women. BMC Womens Health. 2015;15:55

27. Byrom B, Rowe DA. Measuring free-living physical activity in COPD patients: Deriving methodology standards for clinical trials through a review of research studies. Contemp Clin Trials. 2016;47:172-184.

28. Garatachea N, Torres Luque G, Gonzalez Gallego J. Physical activity and energy expenditure measurements using accelerometers in older adults. Nutr Hosp. 2010;25(2):224-230.

29. Santos-Lozano A, Santin-Medeiros F, Cardon G, TorresLuque G, Bailon R, Bergmeir C, Ruiz JR, et al. Actigraph GT3X: validation and determination of physical activity intensity cut points. Int J Sports Med. 2013;34(11):975982.

30. Freedson PS, Melanson E, Sirard J. Calibration of the Computer Science and Applications, Inc. accelerometer. Med Sci Sports Exerc. 1998;30(5):777-781.

31. Sasaki JE, John D, Freedson PS. Validation and comparison of ActiGraph activity monitors. J Sci Med Sport. 2011;14(5):411-416.

32. Butts B, Higgins M, Dunbar S, Reilly C. The third time's a charm: psychometric testing and update of the Atlanta heart failure knowledge test. J Cardiovasc Nurs. 2018;33(1):13-21.

33. Barbaranelli C, Lee CS, Vellone E, Riegel B. Dimensionality and reliability of the self-care of heart failure index scales: further evidence from confirmatory factor analysis. Res Nurs Health. 2014;37(6):524-537.

34. Artinian NT, Magnan M, Sloan M, Lange MP. Self-care behaviors among patients with heart failure. Heart Lung. 2002;31(3):161-172.

35. Akaike H. Information theory and an extension of the maximum likelihood principle. In: Petrov BN, Csaki F (Eds). 2nd International Symposium on Information Theory. Akademia Kiado, Budapest. 1973; p. 267-281.

36. Bentler PM. Comparative fit indexes in structural models. Psychol Bull. 1990;107(2):238-246.

37. Steiger JH. Structural Model Evaluation and Modification: An Interval Estimation Approach. Multivariate Behav Res. 1990;25(2):173-180.

38. Pozehl BJ, Duncan K, Hertzog M, McGuire R, Norman JF, Artinian NT, Keteyian SJ. Study of adherence to exercise in heart failure: the HEART camp trial protocol. BMC Cardiovasc Disord. 2014;14:172.

39. Edwards MK, Loprinzi PD. Sedentary behavior \& healthrelated quality of life among congestive heart failure patients. Int J Cardiol. 2016;220:520-523.

40. Kwak L, Berrigan D, Van Domelen D, Sjostrom M, Hagstromer M. Examining differences in physical activity levels by employment status and/or job activity level: Gender-specific comparisons between the United States and Sweden. J Sci Med Sport. 2016;19(6):482-487.

41. Pulakka A, Stenholm S, Bosma H, Schaper NC, Savelberg H, Stehouwer CDA, van der Kallen CJH, et al. Association between employment status and objectively measured physical activity and sedentary behavior-the Maastricht study. J Occup Environ Med. 2018;60(4):309315.

42. Klocek M, Czarnecka D. [Health-related quality of life in women with chronic heart failure]. Przegl Lek. 2016;73(6):435-438. 